\title{
Intraoperative detection of lymph node micrometastasis with flow cytometry in non-small cell lung cancer
}

\author{
Manabu Ito, MD, Yoshihiro Minamiya, MD, PhD, Hideki Kawai, MD, PhD, Satoshi Saito, Hajime Saito, MD, PhD,
} Kazuhiro Imai, MD, and Jun-ichi Ogawa, MD, PhD

Objective: We sought to determine whether cytokeratin-positive cells can be detected as markers of lymph node metastasis by using flow cytometry within a time frame suitable for intraoperative decision making in non-small cell lung cancer.

Methods: Five lymph nodes from each of 20 patients with non-small cell lung cancer were randomly selected for study. Each node was divided longitudinally into 3 pieces: one piece for flow cytometry, one for immunohistochemical staining, and the last for conventional hematoxylin and eosin staining. In both flow cytometry and immunohistochemistry, cytokeratin-positive cells were detected with the fluorescein isothiocyanate-conjugated anti-cytokeratin antibody AE1/AE3.

Results: Cytokeratin-positive nodes were detected by means of flow cytometry within 40 minutes. Eight (8\%) of the 100 lymph nodes from 4 (20\%) of the 20 patients were deemed positive for metastasis on the basis of conventional histologic examination. By contrast, 33 (33\%) lymph nodes from $13(65 \%)$ patients were deemed positive on the basis of immunohistochemical cytokeratin staining, and 38 (38\%) lymph nodes from $14(70 \%)$ patients were deemed positive on the basis of flow cytometric cytokeratin-positive cell detection. All nodes deemed positive for metastasis on the basis of conventional and immunohistochemical methods were also positive on flow cytometry.

Conclusions: Flow cytometry enables rapid intraoperative diagnosis of nodal metastasis in patients with non-small cell lung cancer. Flow cytometric detection of cytokeratin-positive cells within lymph nodes correlates with their immunohistochemical detection, and its level of sensitivity is greater than that of conventional histologic staining and about equal to that of immunohistochemical staining.

From Akita University School of Medicine, Division of Thoracic Surgery, Department of Surgery, Akita City, Japan.

Received for publication March 22, 2005; revisions received May 3, 2005; accepted for publication May 9, 2005.

Address for reprints: Yoshihiro Minamiya, $\mathrm{MD}, \mathrm{PhD}$, Division of Thoracic Surgery, Department of Surgery, Akita University School of Medicine, 1-1-1 Hondo Akita City 010-8543, Japan (E-mail: minamiya@ med.akita-u.ac.jp).

J Thorac Cardiovasc Surg 2005;130:753-8

$0022-5223 / \$ 30.00$

Copyright () 2005 by The American Association for Thoracic Surgery

doi:10.1016/j.jtcvs.2005.05.012
$\mathrm{W}$ hen a surgeon makes a decision to proceed with further surgical treatment for non-small cell lung cancer (NSCLC; eg, mediastinoscopy and extended segmentectomy), ${ }^{1}$ timely intraoperative determination of the presence or absence of lymph node metastasis is essential. To that end, lymph node metastasis is commonly diagnosed intraoperatively on the basis of an examination of frozen sections. With that technique, however, false-negative results sometimes occur because micrometastasis goes undetected.

Marchevsky and colleagues ${ }^{2}$ reported that patients with NSCLC with isolated tumor cells (ITCs) or micrometastasis in N1 have survival rates similar to those with pN0 disease, which is consistent with findings reported for patients with breast cancer. However, those investigators studied only a limited number of patients over a limited period of time after surgical intervention. By contrast, several other investigators have reported that survival times among patients with micrometastases detected immunohistochemically are no better than those among patients with nodal metastases detected by means of conventional histologic examination..$^{3-7}$ It is therefore generally believed that the presence of nodal ITCs or micrometastasis 
Peripheral leukocytes

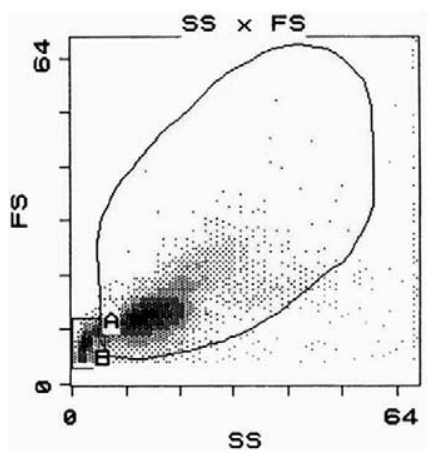

A549 + lymphocytes

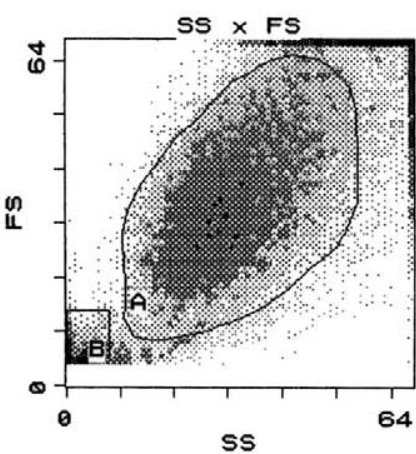

Sq19 + lymphocytes

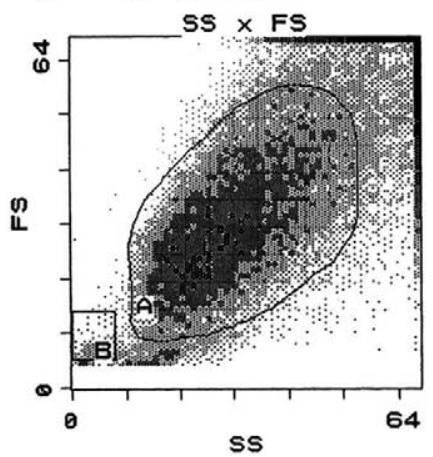

Figure 1. FCM analysis of cultured lung cancer cells. Both A549 and sq19 cancer cells distributed in area A in almost the same pattern, as did primary cancer cells from the lung tumors of our patients. Lymphocytes, by contrast, distributed in area B. FS, Forward scatter; SS, side scatter.

significantly affects the prognosis of patients with NSCLC. This makes it essential that lymph node metastasis is precisely diagnosed and that false-negative results resulting from a failure to detect ITCs or micrometastasis are avoided when making an intraoperative decision about whether to proceed with further surgical treatment.

Techniques that have been applied to accomplish that aim include intraoperative immunohistochemical staining, ${ }^{8-11}$ imprint cytology, ${ }^{12,13}$ and reverse transcriptasepolymerase chain reaction (RT-PCR). ${ }^{14,15}$ None of these methods is problem free, however. For that reason, we have been developing a method that makes use of flow cytometry (FCM) in the diagnosis of nodal metastasis. In the present study we tested whether detection of cytokeratin (CK)positive cells as markers of nodal metastasis with FCM could be carried out within a time frame suitable for intraoperative decision making in NSCLC. We found that FCM enables rapid intraoperative diagnosis of nodal metastasis and that the sensitivity of this method is equal to that of immunohistochemistry.

\section{Materials, Patients, and Methods \\ Patients}

Twenty consecutive patients with NSCLC were enrolled in the study between April 2004 and December 2004 after obtaining signed informed consent. Surgically resected specimens were used under approval of the Institutional Review Boards at Akita University School of Medicine and University Hospital. After a preoperative evaluation, the patients were taken to an operating room, and the standard preparations were made for a thoracotomy, lung resection, and mediastinal lymph node dissection. Five lymph nodes from each patient were randomly selected for study. Each lymph node was divided longitudinally into 3 pieces: one piece was used for FCM, one for immunohistochemical staining, and the last for conventional hematoxylin and eosin staining.

\section{FCM}

The capsule of the dissected lymph node was removed to avoid contamination by normal CK-positive cells derived from lung tissue, after which the cells were dissociated and used to prepare FCM samples with a commercially available kit (IntraPrep Permeabilization Reagent; Coulter cloneImmunotech, Marseille, France). An anti-CK AE1/AE3 antibody cocktail (DAKO Corp, Carpinteria, Calif), which recognizes the 56.5-, 50-, 50'-, 48-, and $40-\mathrm{kd}$ keratins of the acidic subfamily (AE1) and all members of the basic subfamily (AE3), was used to detect CK-positive cells. The anti-CK antibody was preconjugated with fluorescent dye (fluorescein isothiocyanate) by using a protein labeling kit (Molecular Probe, Inc, Eugene, Ore) to avoid the necessity of an incubation step with a secondary antibody. The FCM samples were then incubated with the fluorescently labeled antibody $(5 \mu \mathrm{g} / \mathrm{mL})$ for 15 minutes at room temperature, after which the cells were washed 3 times with phosphate-buffered saline. CK-positive cells were then detected with an FCM Epics Elite (Coulter Electronics, Hialeah, Fla). At least 100,000 cells were analyzed for each lymph node.

\section{Histopathologic Evaluation}

Samples from all dissected lymph nodes were sectioned and conventionally examined with hematoxylin and eosin staining. In addition, other samples were immunohistochemically labeled with anti-CK AE1/AE3 antibody to detect the presence of micrometastases. A result was considered positive if positive cell clusters or individual cells with the appropriate tumor cell morphology were recognized. As proposed by the new American Joint Committee on Cancer Cancer Staging Manual, ${ }^{16}$ ITCs were also considered as positive in this study.

\section{Results}

\section{Detection of CK-Positive Cells With FCM}

We initially characterized the FCM parameters that were subsequently used to identify CK-positive cells. Figure 1 shows the cell distributions obtained when peripheral lym- 
Gate:B (lymphocyte area)
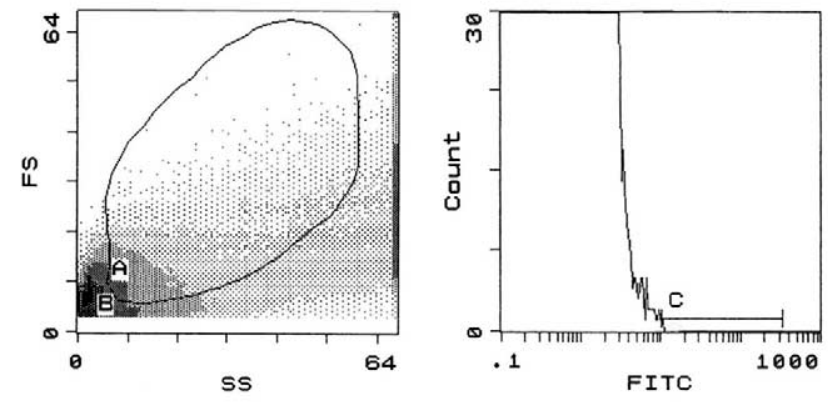

Gate: AC

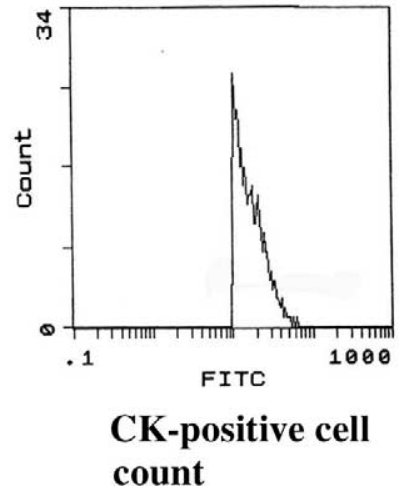

Figure 2. Detection of CK-positive cells within lymph nodes. The distribution of cells from a representative lymph node is depicted in the left panel; note that the gate was set in area A according to the pattern obtained with cultured lung cancer cells (Figure 1). The gate was set in lymphocyte area B to define the CK-negative area because lymphocytes are always CK negative; area C was then defined as the area not included in the lymphocyte area (middle panel). We defined the product of areas $\mathrm{A}$ and $\mathrm{C}$ as the area of CK-positive tumor cells. FS, Forward scatter; SS, side scatter; FITC, fluorescein isothiocyanate.

phocytes alone (left panel) or cultured tumor cells plus lymphocytes (center and right panels) were subjected to FCM. Both the forward scatter and side scatter of the tumor cells were larger than was seen with the lymphocytes, and the distribution of primary tumor cells exhibited virtually the same pattern as the cultured tumor cells (Figure 2, left panel). Gates A (tumor cell area) and B (lymphocyte area) were defined accordingly. The CK-positive area (gate C) was defined, taking the results obtained with gate B (lymphocyte area) into consideration because lymphocytes are always CK negative (Figure 2, middle panel). The product of areas $\mathrm{A}$ and $\mathrm{C}$ (gate $\mathrm{AC}$ ) was then used to define the distribution pattern of CK-positive tumor cells (Figure 2, right panel).

To confirm the accuracy of the FCM detection of CKpositive cells, we correlated known numbers of cultured A549 tumor cells with the numbers of CK-positive cells counted with FCM (Figure 3). The highly significant $(r=$ $0.99999, P<.0001)$ correlation obtained clearly shows that we were able to accurately count CK-positive tumor cells with FCM.

\section{Clinical Evaluation of Metastasis With FCM}

The clinical characteristics of the study participants are summarized in Table 1. CK-positive nodes were detected intraoperatively within about 40 minutes by using FCM and the protocol outlined in Table 2. To define the threshold number of CK-positive cells needed for a lymph node to be deemed metastasis positive, the FCM counts were plotted as in Figure 4, after which the values were compared with those obtained from nodes found to be immunohistochemically CK positive (eg, Figure 5) or CK negative. Using this plot, we defined $0.35 \%$ as the threshold value needed for a diagnosis of metastasis. At that value, there are no false-negative results and only a minimal number of false-positive results.

Table 3 summarizes our clinical results. Eight (8\%) lymph nodes from 4 (20\%) patients were deemed positive

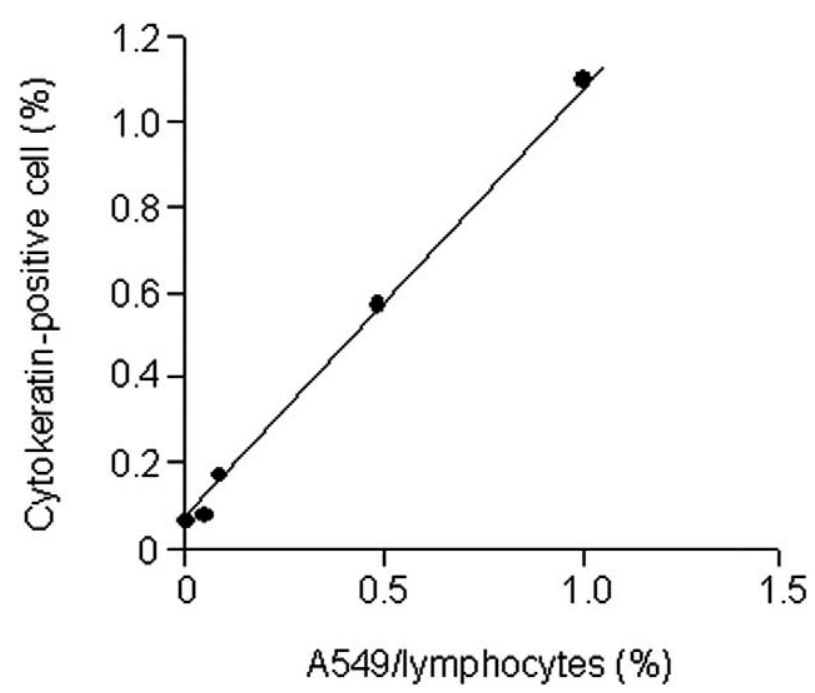

Figure 3. Various numbers of A549 cells were diluted with lymphocytes, after which the size of the CK-positive cell fraction determined with FCM was plotted against the known A549 cell fraction. The very strong correlation $(r=0.99999, P<.0001)$ is indicative of our ability to accurately detect and count CKpositive cells with FCM. 
TABLE 1. Patient characteristics

\begin{tabular}{lc}
\hline Patients & 20 \\
Age (y) & $71.5 \pm 7.0$ \\
Mean & $56-81$ \\
Range & Male: 14 \\
Sex & Female: 6 \\
Pathologic stage & \\
Stage IA & 6 \\
Stage IB & 5 \\
Stage II & 1 \\
Stage IIIA & 4 \\
Stage IIIB & 1 \\
Stage IV & 3 \\
Tumor location & \\
RUL & 7 \\
RML & 1 \\
RLL & 4 \\
LUL & 3 \\
LLL & 5 \\
Tumor size (mm) & \\
Mean & $26.8 \pm 13.6$ \\
Range & $10-60$ \\
Histologic type & \\
Ad & 12 \\
Sq & 8 \\
\hline RUL Right upper & \\
\hline
\end{tabular}

$R U L$, Right upper lobe; $R M L$, right middle lobe; $R L L$, right lower lobe $L U L$, left upper lobe, $L L L$, left lower lobe; ad, adenocarcinoma; $s q$, squamous cell carcinoma.

on the basis of conventional histologic examination. By contrast, 33 (33\%) lymph nodes from $13(65 \%)$ patients were deemed positive on the basis of immunohistochemical CK staining, and 38 (38\%) nodes from 14 (70\%) patients were deemed positive on the basis of FCM CK-positive cell detection. All nodes deemed positive by using conventional and immunohistochemical methods were also positive on FCM. If results of the immunohistochemistry might be correct, the sensitivity, specificity, and accuracy of FCM would be 100\% (33/33), 91.2\% (62/68), and 96.0\% (96/ $100)$, respectively.

TABLE 2. Timetable of cytokeratin-positive cell detection with FCM

\begin{tabular}{ll}
\hline \multicolumn{1}{c}{ Time } & \multicolumn{1}{c}{ Procedure } \\
\hline $5 \mathrm{~min}$ & $\begin{array}{l}\text { Preparation of lymph node single-cell suspension } \\
\text { Hemolysis and cell permeabilization }\end{array}$ \\
$10 \mathrm{~min}$ & $\begin{array}{l}\text { Immunofluorescent staining with antibody AE1/ } \\
\text { AE3 }\end{array}$ \\
$5 \mathrm{~min}$ & FCM \\
Total $=$ & \\
$40 \mathrm{~min}$ & \\
\hline
\end{tabular}

FCM, Flow cytometry.

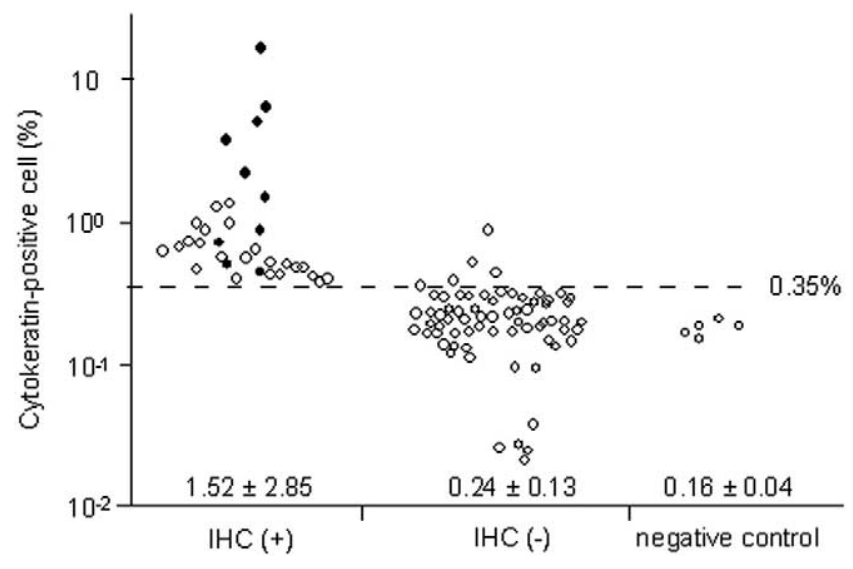

Figure 4. Threshold value for FCM detection of micrometastasis. The percentages of CK-positive cells were plotted against the percentages of immunohistochemically positive $(\mathrm{IHC}[+])$ and negative (IHC[-]) cells. Filled and open circles depict hematoxylin and eosin-positive and hematoxylin and eosin-negative staining, respectively. The threshold line was drawn below the immunohistochemically positive cells at $0.35 \%$. On the basis of this plot, we deemed micrometastasis to be present when the percentage of CK-positive cells determined by means of FCM was more than $0.35 \%$.

\section{Discussion}

The present findings suggest (1) that FCM can be used to rapidly identify CK-positive lymph nodes in patients with

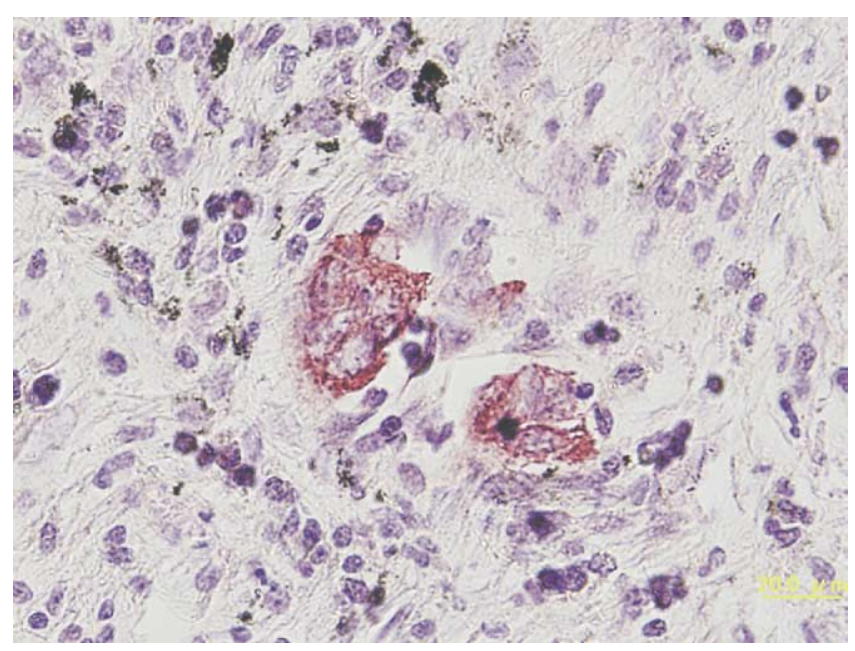

Figure 5. Detection of micrometastasis with CK immunohistochemistry. After labeling lymph nodes with the anti-CK antibody AE1/AE3, the result was considered positive if individual cells or clusters of cells with the appropriate tumor cell morphology were recognized. As proposed by the new American Joint Committee on Cancer Cancer Staging Manual, isolated tumor cells were also diagnosed as metastasis. 
TABLE 3. Diagnosis of micrometastasis with FCM, IHC, and HE

\begin{tabular}{lcc}
\hline Methods & $\mathbf{N}^{+}$patient & $\mathbf{N}^{+}$node \\
\hline FCM & $14 / 20(70 \%)$ & $38 / 100(38 \%)$ \\
IHC & $13 / 20(65 \%)$ & $33 / 100(33 \%)$ \\
HE & $4 / 20(20 \%)$ & $8 / 100(8 \%)$ \\
\hline
\end{tabular}

FCM, Flow cytometry; IHC, immunohistochemistry; $H E$, hematoxylin and eosin.

NSCLC, (2) that detection of CK-positive cells with FCM correlates with immunohistochemical detection, and (3) that FCM is a more sensitive tool for detecting nodal metastasis than conventional histologic evaluation, with a level of sensitivity about equal to that of immunohistochemical examination. It thus appears that we might be able to apply FCM detection of CK-positive cells for intraoperative diagnosis of nodal metastasis in NSCLC. However, the proposed threshold value $(0.35 \%$, Figure 4$)$ can be applied only to our FCM. If other investigators will follow our method, the cut-off value for each FCM must be defined by using true-negative lymph nodes from patients with benign disease (negative control) and true-positive lymph nodes from patients with lung cancer (positive control). For standardization and maintenance of the protocol, the setting of each FCM must be sometimes adjusted with negative and positive controls.

Conventional analysis of frozen tissue sections enables very rapid diagnosis, within 20 minutes at most institutions, ${ }^{17}$ but the sensitivity of this method for detecting micrometastasis is comparatively low. By contrast, the sensitivity of RT-PCR assays, which have been applied to the diagnosis of lymph node metastasis outside the surgical setting, is very high. ${ }^{14,15}$ Unfortunately, the amount of time commonly required to conduct such assays makes them unsuitable for intraoperative decision making. Several investigators have been trying to reduce the time required for RT-PCR assays, however. For instance, Raja and associates ${ }^{18}$ have developed a rapid and quantitative RT-PCR assay that they reported could be used to detect micrometastasis within about 30 minutes. This approach addresses the problem of time, but it does not address a second potential problem: because of its very high sensitivity, conventional RT-PCR produces a comparatively high number of false-positive reactions. ${ }^{19}$

Although immunohistochemical staining also commonly requires several hours to complete, several recently described techniques enable immunohistochemical staining to be accomplished within only 12 to 30 minutes. ${ }^{8-11}$ In addition, imprint cytology is sometimes applied for the diagnosis of malignancy and nodal metastasis because it is convenient and can be done quickly. But although the accuracy of imprint cytology is often sufficient to warrant its use for intraoperative assessment of nodal metastasis instead of frozen sections, ${ }^{20}$ it is not sufficiently sensitive to detect micrometastases. $^{21,22}$ To increase its sensitivity, however, Munakata and coworkers ${ }^{12}$ combined imprint cytology with immunofluorescent labeling of $\mathrm{CK}$ and obtained results similar to those obtained with immunohistochemistry alone. Thus both of these techniques are potentially applicable for intraoperative diagnosis of nodal metastasis and decision making.

In the present study we describe an additional new method for the intraoperative diagnosis of nodal micrometastasis. We were able to detect CK-positive nodes within 40 minutes using FCM, which is within about the same time frame as the aforementioned methods. To further confirm the feasibility of CK detection with FCM for the diagnosis of micrometastasis, in a future investigation, we will compare the FCM method with other rapid methods for detecting micrometastasis.

The use of FCM to detect metastasis was first reported by Joensuu and colleagues, ${ }^{23}$ who used DNA aneuploidy as an indicator of metastasis. In the present study we detected the presence of CK-positive cells within lymph nodes as an indicator of micrometastasis, which means that we need to consider the possibility of false-positive results very carefully. In many instances microscopic evaluation of immunohistochemically stained sections has revealed CK-positive interstitial reticulum cells, sometimes leading to false-positive staining of sinus lining cells. ${ }^{24}$ Although such staining is easily recognized morphologically, no morphologic checks can be made to control for false-positive CK staining when using the FCM method. To avoid these cells, Leers and associ$\operatorname{ates}^{25}$ combined multiparameter FCM detection of metastasis with CK staining and DNA aneuploidy detection in formalin-fixed, paraffin-embedded lymph nodes. We did not stain DNA in our present study because our final goal is to be able to provide the surgeon with this critical information within a time frame that enables important surgical decisions to be made intraoperatively. Instead, we found that nontumorous CK-positive cells could be omitted by using an appropriate threshold value that led to no false-negative results and a minimal number of false-positive results (Figure 4). If results of the immunohistochemistry were correct, the false-positive rate would be $8.1 \%$ (5/67). The importance of false-positive or false-negative findings and their effect on patient care will depend on the treatment offered on the basis of this finding. If detection of micrometastases will mandate that the surgeon performs a complete lymph node dissection versus a node sampling, then greater importance should be placed on minimizing false-negative results. On the other hand, if the patient will be denied or offered a limited resection on the basis of the presence of a positive 
lymph node, then no false-positive results should be accepted. To confirm this finding, we intend to compare our FCM method with multiparameter FCM detection in a future study.

In summary, we have developed a rapid intraoperative FCM technique for the diagnosis of lymph node metastasis. This method appears to be sensitive enough to detect micrometastases, although only a limited number of patients were investigated. Consequently, further investigation will be required to confirm the feasibility of this method.

\section{References}

1. Yoshikawa K, Tsubota N, Kodama K, Ayabe H, Taki T, Mori T. Prospective study of extended segmentectomy for small lung tumors: the final report. Ann Thorac Surg. 2002;73:1055-9.

2. Marchevsky AM, Qiao JH, Krajisnik S, Mirocha JM, McKenna RJ. The prognostic significance of intranodal isolated tumor cells and micrometastases in patients with non-small cell carcinoma of the lung. J Thorac Cardiovasc Surg. 2003;126:551-7.

3. Coello MC, Luketich JD, Litle VR, Godfrey TE. Prognostic significance of micrometastasis in non-small-cell lung cancer. Clin Lung Cancer. 2004;5:214-25.

4. Gu CD, Osaki T, Oyama T, Inoue M, Kodate M, Dobashi K, et al. Detection of micrometastatic tumor cells in pNO lymph nodes of patients with completely resected nonsmall cell lung cancer: impact on recurrence and survival. Ann Surg. 2002;235:133-9.

5. Osaki T, Oyama T, Inoue M, Gu CD, Kodate M, Aikawa M, et al. Molecular biological markers and micrometastasis in resected nonsmall-cell lung cancer. Prognostic implications. Jpn J Thorac Cardiovasc Surg. 2001;49:545-51.

6. Izbicki JR, Passlick B, Hosch SB, Kubuschock B, Schneider C, Busch $\mathrm{C}$, et al. Mode of spread in the early phase of lymphatic metastasis in non-small-cell lung cancer: significance of nodal micrometastasis. J Thorac Cardiovasc Surg. 1996;112:623-30.

7. Coello MC, Luketich JD, Litle VR, Godfrey TE. Prognostic significance of micrometastasis in non-small-cell lung cancer. Clin Lung Cancer. 2004;5:214-25.

8. Matsumoto M, Natsugoe S, Ishigami S, Uenosono Y, Takao S, Aikou T. Rapid immunohistochemical detection of lymph node micrometastasis during operation for upper gastrointestinal carcinoma. Br J Surg. 2003;90:563-6.

9. Richter T, Nahrig J, Komminoth P, Kowolik J, Werner M. Protocol for ultrarapid immunostaining of frozen sections. J Clin Pathol. 1999;52: 461-3.

10. Eudy GE, Carlson GW, Murray DR, Waldrop SM, Lawson D, Cohen C. Rapid immunohistochemistry of sentinel lymph nodes for metastatic melanoma. Hum Pathol. 2003;34:797-802.

11. Nahrig JM, Richter T, Kuhn W, Avril N, Flatau B, Kowolik J, et al. Intraoperative examination of sentinel lymph nodes by ultrarapid immunohistochemistry. Breast J. 2003;9:277-81.
12. Munakata S, Aihara T, Morino H, Takatsuka Y. Application of immunofluorescence for intraoperative evaluation of sentinel lymph nodes in patients with breast carcinoma. Cancer. 2003;98:1562-8.

13. Clarke MR, Landreneau RJ, Borochovitz D. Intraoperative imprint cytology for evaluation of mediastinal lymphadenopathy. Ann Thorac Surg. 1994;57:1206-10.

14. Iwao K, Watanabe T, Fujiwara Y, Takami K, Kodama K, Higashiyama $\mathrm{M}$, et al. Isolation of a novel human lung-specific gene, LUNX, a potential molecular marker for detection of micrometastasis in nonsmall-cell lung cancer. Int J Cancer. 2001;91:433-7.

15. Matsuda J, Kitagawa Y, Fujii H, Mukai M, Dan K, Kubota T, et al. Significance of metastasis detected by molecular techniques in sentinel nodes of patients with gastrointestinal cancer. Ann Surg Oncol. 2004; 11(suppl):250S-4S.

16. American Joint Committee on Cancer. AJCC cancer staging manual. 6th ed. New York: Springer; 2002.

17. Novis DA, Zarbo RJ. Interinstitutional comparison of frozen section turnaround time. A College of American Pathologists Q-Probes study of 32868 frozen sections in 700 hospitals. Arch Pathol Lab Med. 1997;121:559-67.

18. Raja S, Luketich JD, Kelly LA, Gooding WE, Finkelstein SD, Godfrey TE. Rapid, quantitative reverse transcriptase-polymerase chain reaction: application to intraoperative molecular detection of occult metastases in esophageal cancer. J Thorac Cardiovasc Surg. 2002;123: 475-82.

19. Bostick PJ, Chatterjee S, Chi DD, Huynh KT, Giuliano AE, Cote R, et al. Limitations of specific reverse-transcriptase polymerase chain reaction markers in the detection of metastases in the lymph nodes and blood of breast cancer patients. J Clin Oncol. 1998;16:2632-40.

20. Llatjos M, Castella E, Fraile M, Rull M, Julian FJ, Fuste F, et al. Intraoperative assessment of sentinel lymph nodes in patients with breast carcinoma: accuracy of rapid imprint cytology compared with definitive histologic workup. Cancer. 2002;96:150-6.

21. Turner RR, Hansen NM, Stern SL, Giuliano AE. Intraoperative examination of the sentinel lymph node for breast carcinoma staging. Am J Clin Pathol. 1999;112:627-34.

22. Creager AJ, Geisinger KR, Shiver SA, Perrier ND, Shen P, Ann Shaw $\mathrm{J}$, et al. Intraoperative evaluation of sentinel lymph nodes for metastatic breast carcinoma by imprint cytology. Mod Pathol. 2002;15: 1140-7.

23. Joensuu H, Klemi PJ, Eerola E. Flow cytometric DNA analysis combined with fine needle aspiration biopsy in the diagnosis of palpable metastases. Anal Quant Cytol Histol. 1988;10:256-60.

24. Gould VE, Bloom KJ, Franke WW, Warren WH, Moll R. Increased numbers of cytokeratin-positive interstitial reticulum cells (CIRC) in reactive, inflammatory and neoplastic lymphadenopathies: hyperplasia or induced expression? Virchows Arch. 1995;425:617-29.

25. Leers MP, Schoffelen RH, Hoop JG, Theunissen PH, Oosterhuis JW, vd Bijl H, et al. Multiparameter flow cytometry as a tool for the detection of micrometastatic tumour cells in the sentinel lymph node procedure of patients with breast cancer. J Clin Pathol. 2002;55:35966. 